\title{
PECULIARITIES OF PRE-COMPETITIVE PSYCHOLOGICAL FACTORS, SPORT PERFORMANCE INDICATORS AND PHYSIOLOGICAL DEMANDS IN YOUTH GOLF
}

\author{
Marius Zienius, Antanas Skarbalius, Aurelijus Kazys Zuoza, Kazimieras Pukẻnas \\ Lithuanian Sports University, Kaunas, Lithuania
}

\begin{abstract}
Background. Sport performance indicators, physiological demands and psychological hallmarks are the key factors in the development of golf performance. However, no research was found in youth golf. We hypothesized that the peculiarities of pre-competitive psychological factors, sport performance indicators, physiological demands in youth golf are not adequate compared with adult golf.

Methods. Ten male golfers participated in this study. The aim of the research was to assess and evaluate the peculiarities of pre-competitive psychological factors, sport performance indicators, physiological demands in youth golf. Sport performance indicators in golf were recorded using a personal sport performance statistical protocol. Locomotion and physiological demands were recorded using the FRWD W ${ }^{400}$ Series (Finland) device. Competitive state anxiety was assessed with the Competitive State Anxiety Inventory-2 (CSAI-2). The CSAI-2 assesses two components of state anxiety, cognitive and somatic anxiety, as well as a related construct, self-confidence.

Results. The subjects' long-game skills were at a higher level than their short-game skills. Pre-competitive cognitive and somatic anxiety was low, but self-confidence was high. Training effect on young golfers' aerobic fitness was low (1.28 - recovery zone).

Conclusions. Opposite to elite golfers, the long-game skills of youth golfers are more developed than their short-game skills. Playing golf does not have a positive training effect on young golfers' aerobic fitness. High level of self-confidence in youth golfers, which is adequate to elite golfers, could be determined by the similar level of mastership among competitive participants.
\end{abstract}

Keywords: cognitive and somatic anxiety, self-confidence, long-game skills, short-game skills, aerobic fitness.

\section{INTRODUCTION}

$\mathrm{T}$ The sport performance indicators (Hellström, 2009 a), physiological demands (Smith, 2010) and psychological hallmarks (Hellström, 2009 b) of elite golfers are the key factors when golfers and their coaches compose annual training programmes. Each of these factors are equally important because golf is a game in which a small advantage in one area can mean the difference between finishing the first or finishing the $20^{\text {th }}$ (Draovitch \& Simpson, 2007). Hence, youth golfers need to develop all three factors in order to improve or optimize sport performance in golf (Draovitch \& Simpson, 2007; Hellström, 2009 a; Hellström, 2009 b; Smith, 2010).
The player with the lowest total number of strokes is the winner of the competition and it is usually determined by better short-game skills (chipping, pitching, bunkers shots and putting) (Hellström, 2009 a). The best players on the Professional Golfers' Association (PGA) Tour are more accurate in the short-game than the rest of the players (Pelz, 1999; Dorsel \& Rotunda, 2001). Accurately performed chipping, pitching or a bunker shot will have a significant effect on the number of one-putts (Pelz, 1999, 2000). The ability to hit the ball a long way (driving distance) and to be accurate from tee to fairway (driving accuracy) affects the final score too; from the 
long-game skills, the green in regulation (GIR; see the definitions in Table 1) shows the strongest correlation with the scoring average (Hellström, 2009 a). However, in general, the indicators describing the short-game skills (scrambling and putts per GIR; see the definitions in Table 1) have the strongest correlations with the scoring average (Hellström, 2009 a).

Physiological demands show the physical state of golfers during the play on the golf course and the described peculiarities of the play (Draovitch \& Simpson, 2007; Smith, 2010). For better performance it is necessary that the physiological state of the golfer would remain as close to the starting point as possible without any undue fatigue (Smith, 2010). Golf players who are physically fit can stay focused throughout the game; higher fitness levels also reduce recovery time (Draovitch \& Simpson, 2007). Lower heart rate (HR) before the execution of the shot is one of the factors allowing players to achieve a better outcome (Boutcher \& Zinsser, 1990; Cotterill \& Collins, 2005; Smith, 2010; Neumann \& Thomas, 2011; van der Lei \& Tenenbaum, 2012). Golfers must integrate the physiological aspects of performance into their development plan in order to reach optimal movement capabilities (Smith, 2010).

Elite golfers are so good in technique and physical fitness that psychology usually becomes the leading factor determining a better performance among elite golfers (Hellström, 2009b). The attitude, confidence, ability to handle pressure, desire, concentration and motivation are important psychological qualities necessary to successfully participate in tournaments for elite golfers (Douglas \& Fox, 2002). Meanwhile, the main cause of poor performance is the loss of confidence (Douglas \& Fox, 2002). Moreover, skilled players indicated that the peak performance was related to being focused, feeling of performing effortlessly, automatically, being physically relaxed and mentally calm, feeling in control, having no fear, having high confidence, and experiencing fun and enjoyment (Cohn, 1991). Beauchamp (1999) noted that confidence, imagery and task focus were the most significant psychological skills for the peak putting performance among elite golfers. Differences in personality may explain why mood and emotions have no strong relationships to golf scores and it seems to be individual (Hellström, 2009 b). According to Martens, Vealey, and Burton (1990), less skilled sportspeople report the higher overall levels of anxiety compared with elite competitors because they are less capable of self-regulating thoughts and are more likely to experience worry and self-doubt. Therefore, each player must have coping strategies when the stress arise during the play and these coping skills must be improved consistently (Hellström, 2009 b).

Review articles show that sport performance indicators (Hellström, 2009 a), physiological demands (Smith, 2010) and psychological hallmarks (Hellström, 2009 b) are usually analysed in adult golf. However, until now no peculiarities of these mentioned aspects are known in order to give the suitable training program for youth golfers in the long-term target development. We hypothesized that the peculiarities of pre-competitive psychological factors, sport performance indicators, physiological demands in youth golf were not adequate as compared with adult golf. The aim of the research was to assess and evaluate the peculiarities of pre-competitive psychological factors, sport performance indicators and physiological demands in youth golf.

\section{METHODS}

Participants. Ten male golfers (age $16.7 \pm 1.95$ years, height $180.2 \pm 7.63 \mathrm{~m}$, body mass $69.1 \pm$ $7.77 \mathrm{~kg}$, handicap index $8.14 \pm 3.52$ ) participated in this study. The participants belonged to the Lithuanian national youth golf team. The experimental procedures were performed in accordance with the Declaration of Helsinki. The participants and their parents gave their written informed consent. The Ethics Committee of Kaunas Regional Biomedical Research approved this study.

Instrumentation. Sport performance indicators in golf (Table 1) were recorded using a sport performance personal statistical protocol (Schempp \& Mattsson, 2005). Locomotion and physiological demands (Table 1) were recorded using the FRWD $\mathrm{W}^{400}$ Series (Finland) device, which allowed us to determine locomotion and physiological indicators according to the age, height, body mass and basal heart rate indicators of the participants (PerezTurpin, Cortell-Tormo, Suarez-Llorca, AndreuCabrera, Llana-Belloch, \& Perez-Soriano, 2009). Competitive state anxiety (Table 1) was assessed with the Competitive State Anxiety Inventory-2 (CSAI-2; Martens, Burton, Vealey, Bump, \& Smith, 1990), which is a self-report psychometric 
inventory of state anxiety consisting of 27 items. The CSAI-2 assesses two components of state anxiety, cognitive and somatic anxiety, and a related construct, self-confidence, with 9 items representing each subscale. The CSAI-2 has good reliability and validity and has been widely used in the research on anxiety in sport (Martens et al., 1990). Each CSAI-2 subscale is rated on a scale from 1 = "not at all" to 4 = "very much so", relating to the extremes of intensity. Thus, each subscale has a range from 9 to 36. Higher scores of cognitive and somatic anxiety indicate higher levels of anxiety, whereas higher scores of the selfconfidence sub-scale correspond to higher levels of self-confidence.

Research design. The study was conducted over five days (August 10-14) at the Capitals Golf Club, Lithuania (par 71; yellow tees $5750 \mathrm{~m}$ ), a course that has moderate elevation changes.

The participants were divided into five pairs of two players. Each pair played only one golf round (18 holes) in the golf course starting at 12:00 and each pair played this round in separate days in the same conditions of play: yellow tees, with a caddy (a person who carries a player's bag and clubs); sunshine and light breeze, and the average temperature was $20^{\circ} \mathrm{C}$.

Fifteen minutes prior to the golf round, the participants completed the psychometric measure of competitive state anxiety (CSAI-2). Later, the global positioning system device (FRWD W $\mathrm{W}^{400}$ Series) was attached to the upper right arm of the subjects to record locomotion indicators. After 1-2 minutes, a signal from the device indicated its connection with the satellite. The pulsometer (FRWD W ${ }^{400}$ Series) was also attached to the chest of the subjects. The recording of locomotion and physiological indicators was started by the subject pressing a button on the device when teeing up at the 1st hole and continued until the play finished with the final putt at the 18th hole when the device was turned off. During the play, the players recorded sport performance indicators; personal statistical protocols of sport performance were recorded after each player completed a hole (18 times). It should be noted that the procedure of completing CSAI2, recording sport performance protocol and using the FRWD W ${ }^{400}$ Series (Finland) device was usual for the golfers because they previously applied it in the training and competition environment.

Statistical analysis. The descriptive statistics of data were presented as the mean and standard deviation (SD). The relationship between the sport performance indicators (driving accuracy, green in regulation, scrambling, putts per green in regulation, putts per round) and the score (strokes per round) was estimated using Pearson's correlation coefficient. The relationship between the pre-competitive psychological factors (cognitive and somatic anxiety, self-confidence) and the sport performance indicators (score, driving accuracy, green in regulation, scrambling, putts per green in regulation, putts per round) was estimated applying Spearman's correlation coefficient. The level of significance was set a priori at $\mathrm{p}<0.05$ for all analyses. The statistical analysis was carried out using SPSS 21.0 package for Windows.

Table 1. Sport Performance Indicators, Locomotion and Physiological Demands, Pre-Competitive Psychological Factors

\begin{tabular}{|c|c|}
\hline $\begin{array}{l}\text { Sport performance } \\
\text { indicators }\end{array}$ & $\begin{array}{l}\text { Driving accuracy (DA) }(\%) \text {; green in regulation (GIR) }(\%) \text {; scrambling }(\%) \text {; putts per GIR; putts per } \\
\text { round; score (holes } 1-9 ; 10-18 ; 1-18) \text { (strokes). }\end{array}$ \\
\hline Locomotion demands & Duration of play (min); distance covered $(\mathrm{m})$; distance covered in excess of course distance $(\mathrm{m}$ and \%). \\
\hline $\begin{array}{l}\text { Physiological } \\
\text { demands }\end{array}$ & $\begin{array}{l}\text { Heart rate }(\mathrm{HR})\left(\text { beats } \cdot \mathrm{min}^{-1}\right) \text {; maximal heart rate }(\mathrm{HRmax})\left(\text { beats } \cdot \mathrm{min}^{-1}\right) \text {; total energy expenditure } \\
(\mathrm{kcal}) \text {; energy expenditure per minute }\left(\mathrm{kcal} \cdot \mathrm{min}^{-1}\right) \text {; maximal energy expenditure per minute }\left(\mathrm{kcal} \cdot \mathrm{min}^{-1}\right) \text {; } \\
\text { maximal excess post-exercise oxygen consumption }(\mathrm{EPOCmax})\left(\mathrm{mL} \cdot \mathrm{kg}^{-1}\right) \text {; maximal oxygen uptake } \\
\left(\mathrm{VO}_{2} \mathrm{max}\right)\left(\mathrm{mL} \cdot \mathrm{kg}^{-1} \cdot \mathrm{min}^{-1}\right) \text {; maximal ventilatory response }\left(\mathrm{L} \cdot \mathrm{min}^{-1}\right) ; \text { maximal respiration rate } \\
\left.\text { (breaths } \cdot \mathrm{min}^{-1}\right) \text {; training effect }(\mathrm{TE})(\mathrm{scale}, 1-5) \text {. }\end{array}$ \\
\hline Psychological factors & Cognitive and somatic anxiety; self-confidence. \\
\hline
\end{tabular}

Notes. Driving accuracy (DA), the percentage of times a player hits the fairway with the tee shot on par 4 and par 5 holes (regardless of club). Green in regulation (GIR), the percentage of times a player hits the green in regulation (greens hit in regulation/holes played); a green is considered hit in regulation if any portion of the ball touches the putting surface and the number of strokes taken is two or less than par. Scrambling, the percentage of times a player misses the GIR, but still makes par or better. Putts per GIR, the average number of putts per GIR. Par, a hole is classified by its par, meaning the number of strokes a skilled golfer should require to complete play of the hole; a hole is either par 3, 4 or 5. Training effect (TE), this indicates how a work-out improves aerobic (endurance) fitness; TE has five levels (1-1.9- easy recovery; 2.0-2.9 maintaining fitness; 3.0-3.9 improving fitness; 4.0-4.9 highly improving; 5.0 - overreaching). 


\section{RESULTS}

The subjects' long-game skills (DA, GIR) were at a higher level than their short-game skills (scrambling; putts per GIR; putts per round) (Table 2). The scoring average was $82.4 \pm 5.93$ strokes (front nine holes $-41.8 \pm 2.74$ strokes; back nine holes $-40.6 \pm 3.5$ strokes). From sport performance indicators, GIR had the strongest relationship to the score (strokes per round) $(\mathrm{r}=-.716 ; \mathrm{p}<.05)$.

The subjects played 3 hours and 45 minutes on average, covering slightly more than $9 \mathrm{~km}$; that is, they exceeded the course distance off the yellow tees by $58 \%$ (Table 3 ).

The results confirmed that the physiological demands arising from golf were not considered intense (Table 4).

Pre-competitive cognitive and somatic anxiety was low, but self-confidence was high (Table 5). Self-confidence had the strongest relationships to the DA percentage $(r=.669 ; \mathrm{p}<0.05)$ and the score (strokes per round) $(\mathrm{r}=-.539)$. While, cognitive anxiety had the strongest relationship to putts per GIR $(r=.346)$. Somatic anxiety had the strongest relationship to scrambling $(r=-.319)$.

\begin{tabular}{|c|c|c|c|c|}
\hline DA (\%) & GIR (\%) & Scrambling (\%) & Putts per GIR & Putts per round \\
\hline $59.23 \pm 10.29$ & $47.22 \pm 11.19$ & $27.86 \pm 16.44$ & $2.09 \pm 0.18$ & $34.6 \pm 2.59$ \\
\hline
\end{tabular}

Table 2. Sport Performance Indicators of Youth Golfers

\begin{tabular}{|c|c|c|c|}
\hline \multirow{2}{*}{$\begin{array}{c}\text { Playing } \\
\text { duration (min) }\end{array}$} & Covered distance (m) & Distance covered in excess of course distance \\
\cline { 3 - 4 } & & $\mathbf{m}$ & $\%$ \\
\hline $224.6 \pm 9.7$ & $9098.2 \pm 171.47$ & $3348.2 \pm 171.47$ & $58.23 \pm 2.98$ \\
\hline
\end{tabular}

Table 3. Locomotion Demands of Youth Golfers

\begin{tabular}{|c|c|c|c|c|}
\hline $\begin{array}{c}\text { HR } \\
\text { (beats· } \min ^{-1} \text { ) }\end{array}$ & $\begin{array}{c}\text { HRmax } \\
\left(\text { beats } \cdot \min ^{-1}\right)\end{array}$ & $\begin{array}{c}\text { Total energy } \\
\text { expenditure (kcal) }\end{array}$ & $\begin{array}{c}\text { Energy } \\
\text { expenditure per } \\
\text { minute } \\
\left(\mathrm{kcal} \cdot \mathrm{min}^{-1}\right) \\
\end{array}$ & $\begin{array}{c}\text { Max. energy } \\
\text { expenditure per } \\
\text { minute } \\
\left(\mathrm{kcal} \cdot \mathrm{min}^{-1}\right)\end{array}$ \\
\hline $108.6 \pm 5.89$ & $147.3 \pm 5.23$ & $1084.1 \pm 134.37$ & $4.82 \pm 0.53$ & $12.9 \pm 1.1$ \\
\hline $\begin{array}{c}\text { EPOCmax } \\
\left(\mathrm{mL} \cdot \mathrm{kg}^{-1}\right)\end{array}$ & $\begin{array}{c}\mathrm{VO}_{2} \mathrm{max} \\
\left(\mathrm{mL} \cdot \mathrm{kg}^{-1} \cdot \mathrm{min}^{-1}\right)\end{array}$ & $\begin{array}{l}\text { Max. ventilatory } \\
\text { response }\left(\mathrm{L} \cdot \mathrm{min}^{-1}\right)\end{array}$ & $\begin{array}{c}\text { Max. respiration } \\
\text { rate } \\
\left(\text { breaths } \cdot \mathbf{m i n}^{-1}\right)\end{array}$ & $\begin{array}{l}\text { Training effect } \\
\text { (scale, } 1-5)\end{array}$ \\
\hline $6 \pm 1.83$ & $37.7 \pm 3.62$ & $73.8 \pm 10.41$ & $44.9 \pm 5.2$ & $1.28 \pm 0.15$ \\
\hline
\end{tabular}

Table 4. Physiological Demands of Youth Golfers

\begin{tabular}{|c|c|c|}
\hline $\begin{array}{c}\text { Cognitive anxiety } \\
\text { (scores) }\end{array}$ & $\begin{array}{c}\text { Somatic anxiety } \\
\text { (scores) }\end{array}$ & $\begin{array}{c}\text { Self-confidence } \\
\text { (scores) }\end{array}$ \\
\hline $16.1 \pm 2.38$ & $13.9 \pm 2.13$ & $29 \pm 2.54$ \\
\hline
\end{tabular}

Table 5. Pre-Competitive Cognitive and Somatic Anxiety, SelfConfidence of Youth Golfers

\section{DISCUSSION}

The subjects' sport performance indicators were very different compared with those of professional golf players in the season of 2013. The clearest difference relates to short-game skills where the Professional Golfers' Association (PGA) Tour players recorded 29.19 putts per round, 1.78 putts per GIR and $57.79 \%$ for scrambling (http:// www.pgatour.com). The subjects performed 5.41 putts per round on average and 0.31 putts per GIR more than the PGA Tour players. Furthermore, the scrambling percentage of the subjects was on average $30 \%$ less than that for the PGA Tour players. Scrambling is currently the best general measure for the short game skills, which involves 
the players' abilities around the green and on the putting green (Hellström, 2009 a). Meanwhile, the difference between the long-game skills of the subjects and professional golf players was less. The green in regulation percentage of the subjects was $47.22 \%$ on average, somewhat lower than the $64.99 \%$ for the professional players (http://www. pgatour.com). GIR is currently the best measure for the long-game skills (Hellström, 2009 a). The percentage for the driving accuracy of the subjects was $59.23 \%$ on average, which was slightly lower compared with the performance of the best players in the world (61.29\%) (http://www.pgatour.com). In summary, it is clear that the subjects' long-game skills (DA, GIR) were at a higher level than their short-game skills (scrambling; putts per GIR; putts per round). The researchers (Pelz, 1999, 2000; Dorsel \& Rotunda, 2001; Hellström, 2009 a) noted that better short-game skills were directly related to better outcome; the best players in the world distinguish themselves from the rest of the players by the short-game skills. Hence, in the future, it is necessary for junior golfers to improve their short-game skills because the indicators describing these skills have the strongest correlations with the scoring average (Hellström, 2009 a). In our case, the subjects' GIR percentage was found as the most important for a better score (strokes per round) $(r=-.716 ; \mathrm{p}<.05)$. The researchers found that scrambling $(\mathrm{r}=-.67 ; \mathrm{p}<.05)$ (Finley $\&$ Halsey, 2004) and putts per GIR $(r=.63 ; \mathrm{p}<$ .05) (Quinn, 2006) had the strongest correlations with the scoring average in elite golf, but GIR also was an indicator strongly related to the low scoring average (Finley \& Halsey, 2004; Quinn, 2006).

The locomotion and physiological demands of subjects were in line with the findings of other studies. A single round of golf can last from 2.5 to $6 \mathrm{~h}$ (Smith, 2010); the distance covered is generally less than $10 \mathrm{~km}$ (Kobriger, Smith, Hollman, \& Smith, 2006; Zunzer, von Duvillard, Tschakert, Mangus, \& Hofmann, 2013). The HR response range is reported as $100-120$ beats. $\mathrm{min}^{-1}$ (McKay, Selig, Carlson, \& Morris, 1997; Burkett \& von HeijneFisher, 1998; Stauch, Lui, Giesler, \& Lehmann, 1999); the range for total energy expenditure is 960-1954 kcal and that for energy expenditure is 6.0-11.8 $\mathrm{kcal} \cdot \mathrm{min}^{-1}$ (Smith, 2010). Our additional findings suggest that playing golf did not have a positive training effect on the subjects' aerobic fitness (1.28 - recovery zone). Hence, playing golf, unfortunately, is not the best means for getting in a better shape to play a better golf. Draovitch and Simpson (2007) noted that golf players were likely to suffer from a performance decrement unless they had a moderately high level of aerobic fitness. It's highly important that the golfer's aerobic state was optimal during the shot preparation and execution, which enables the players to achieve a better outcome (Boutcher \& Zinsser, 1990; Cotterill \& Collins, 2005; Smith, 2010; Neumann \& Thomas, 2011; van der Lei \& Tenenbaum, 2012). Considering the player's optimization from a multidisciplinary perspective, the role of physiology should be seen as equally important as other contributing factors (Smith, 2010). Therefore, young golfers should be advised to include aerobic training sessions in their training programmes with the aim of improving sport performance.

The subjects' pre-competitive cognitive and somatic anxiety was low. Meanwhile, high level of self-confidence was among all players. High self-confidence is one of the most significant psychological factors in golf which allows the players to compete successfully (Cohn, 1991; Beauchamp 1999; Douglas \& Fox, 2002; Hellström, 2009 b). Being mentally calm is also a very significant factor for a better performance (Cohn, 1991; Hellström, 2009 b). The research results are relatively in line with McKay (1998) who noted that pre-competitive cognitive anxiety (scores - 17.1 and 15.1) of elite and amateur golfers was higher than somatic anxiety (scores - 13.9 and 13.5); while self-confidence was moderately high (scores -25.9 and 22.8). Furthermore, McKay (1998) determined that the cognitive (scores - 13.7 and 12.9) and somatic (scores - 11.2 and 10.7) anxiety of elite and amateur golfers before the practice round was significantly lower $(\mathrm{p}<.05)$, while their selfconfidence (scores -28.1 and 26.4) was significantly higher $(\mathrm{p}<.05)$ than that before the competition. Weinberg \& Genuchi (1980) revealed that golfers with lower pre-competitive anxiety performed better than those with higher anxiety levels. The subjects' self-confidence was higher compared with that of elite and amateur golfers (McKay, 1998) and it is a feature which probably allows the subjects to compete more successfully (Cohn, 1991; Beauchamp, 1999; Douglas \& Fox, 2002; Hellström, 2009 b). However, pre-competitive cognitive anxiety of subjects should be lower (McKay, 1998), being mentally calm influences a better execution (Cohn, 1991; Hellström, 2009 b). Furthermore, the subjects' self-confidence levels 
had the strongest influence on the DA percentage $(\mathrm{r}=.669 ; \mathrm{p}<.05)$ and the score (strokes per round) $(\mathrm{r}=-.539)$. McKay (1998) observed that the self-confidence levels of amateur players also had influence on the score (strokes per round) $(\mathrm{r}=-.37)$. Meanwhile, the subjects' cognitive anxiety levels had the strongest influence on the number of putts per GIR $(r=.346)$.

\section{CONCLUSIONS}

The long-game skills of youth golfers are more developed than their short-game skills, which is opposite to elite golfers. Secondly, the moderate-high levels of aerobic fitness are essential for the golfers to compete successfully; however, there is evidence that playing golf does not have a positive training effect on the young golfers' aerobic fitness. The findings suggest that the high level of self-confidence in youth golfers, which is adequate to that of elite golfers, could be determined by the similar level of mastership among competitive participants. The research results allow practitioners to optimize the training programmes of the target group of youth golfers.

Further research is needed for determining the relationships between sport performance, fitness and psychological aspects in youth golf.

\section{REFERENCES}

Beauchamp, P. H. (1999). Peak putting performance: Psychological skills and strategies utilized by PGA Tour golfers. In M. R. Farrally, A. J. Cochran (Eds.), Science and Golf III: Proceedings of the World Scientific Congress of Golf (pp. 181-189). Champaign, Illinois: Human Kinetics.

Boutcher, S. H., \& Zinsser, N. W. (1990). Cardiac deceleration of elite and beginning golfers during putting. Journal of Sport and Exercise Psychology, 12(1), 37-47.

Burkett, L. N., \& von Heijne-Fisher, U. (1998). Heart rate and calorie expenditure of golfers carrying their clubs and walking flat and hilly golf courses. International Sports Journal, 2(2), 78-85.

Cohn, P. J. (1991). An exploratory study on peak performance in golf. The Sport Psychologist, 5(1), 1-14.

Cotterill, S., \& Collins, D. (2005). Heart rate deceleration characteristics across shot types in golf. Journal of Sports Sciences, 23(2), 173-174.

Dorsel, T. N., \& Rotunda, R. J. (2001). Low scores, top 10 finishes, and big money: An analysis of Professional Golf Association Tour statistics and how these relate to overall performance. Perceptual and Motor Skills, 92(2), 575-585. PMID: 11361324 [PubMed - indexed for MEDLINE]

Douglas, K., \& Fox, K. R. (2002). Practice for competition in women professional golfers. In E. Thain (Ed.), Science and Golf IV: Proceedings of the World Scientific Congress of Golf (pp. 257-267). New York: Routledge.

Draovitch, P., \& Simpson, R. (2007). Complete conditioning for golf. Champaign, Illinois: Human Kinetics.

Finley, P. S., \& Halsey, J. J. (2004). Determinants of PGA Tour success: An examination of relationships among performance, scoring, and earnings. Perceptual and Motor Skills, 98(3), 1100-1106. PMID: 15209327

[PubMed - indexed for MEDLINE]

Hellström, J. (2009 a). Competitive elite golf. Sports Medicine, 39(9), 723-741. doi: 10.2165/11315200000000000-00000

Hellström, J. (2009 b). Psychological hallmarks of skilled golfers. Sports Medicine, 39(10), 845-855. doi: 10.2165/11317760-000000000-00000

Kobriger, S. L., Smith, J., Hollman, J. H., \& Smith, A. M. (2006). The contribution of golf to daily physical activity recommendations: How many steps does it take to complete a round of golf? Mayo Clinic Proceedings (Mayo Foundation for Medical Education and Research), 81(8), 1041-1043. PMID: 16901027 [PubMed - indexed for MEDLINE]

van der Lei, H., \& Tenenbaum, G. (2012). Performance processes within affect-related performance zones: A multi-modal investigation of golf performance. Applied Psychophysiology and Biofeedback, 37(4), 229-240. doi: 10.1007/s10484-012-9195-0

Martens, R., Burton, D., Vealey, R. S., Bump, L. A., Smith, D. E. (1990). The competitive state-anxiety inventory-2 (CSAI-2). In R. Martens, R. S. Vealey, D. Burton (Eds.), Competitive anxiety in sport (pp. 117190). Champaign, Illinois: Human Kinetics.

Martens, R., Vealey, R. S., \& Burton, D. (1990). Competitive anxiety in sport. Champaign, Illinois: Human Kinetics.

McKay, J. M. (1998). An investigation of psychophysiological stress in competitive golf. Doctoral dissertation, Victoria University of Technology.

McKay, J. M., Selig, S. E., Carlson, J. S., \& Morris, T. (1997). Psychophysiological stress in elite golfers during practice and competition. Australian Journal of Science and Medicine in Sport, 29(2), 55-61. PMID: 9242979 [PubMed - indexed for MEDLINE] 
Neumann, D. L., \& Thomas, P. R. (2011). Cardiac and respiratory activity and golf putting performance under attentional focus instructions. Psychology of Sport and Exercise, 12(4), 451-459.

Pelz, D. (2000). Dave Pelz's putting bible. New York: Random House.

Pelz, D. (1999). The short game bible. New York: Broadway Books.

Perez-Turpin, J. A., Cortell-Tormo, J. M., SuarezLlorca, C., Andreu-Cabrera, E., Llana-Belloch, S., \& Perez-Soriano, P. (2009). Relationship between anthropometric parameters, physiological responses, routes and competition results in formula windsurfing. Acta Kinesiologiae Universitatis Tartuensis, 14, 95-110.

Quinn, R. J. (2006). Exploring correlation coefficients with golf statistics. Teaching Statistics, 28(1), 10-13. doi: 10.1111/j.1467-9639.2006.00229.x

Schempp, P., \& Mattsson, P. (2005). Golf: Steps to success. Champaign, Illinois: Human Kinetics.
Smith, M. (2010). The role of physiology in the development of golf performance. Sports Medicine, 40(8), 635-655. doi: 10.2165/11532920-00000000000000

Stauch, M., Lui, Y., Giesler, M., \& Lehmann, M. (1999). Physical activity levels during a round of golf on a hilly course. Journal of Sports Medicine and Physical Fitness, 39(4), 321-327. PMID: 10726433 [PubMedindexed for MEDLINE]

Weinberg, R. S., \& Genuchi, M. (1980). Relationship between competitive trait anxiety, state anxiety, and golf performance: A field study. Journal of Sport Psychology, 2, 148-154.

Zunzer, S. C., von Duvillard, S. P., Tschakert, G., Mangus, B., \& Hofmann, P. (2013). Energy expenditure and sex differences of golf playing. Journal of Sports Sciences, 31(10), 1045-1053. doi: $10.1080 / 02640414.2013 .764465$
Corresponding author Marius Zienius Lithuanian Sports University Sporto str. 6, LT-44221 Kaunas Lithuania Tel +370 61346179,

E-mailmariuszienius@yahoo.com 